\title{
More on the metric projection onto a closed convex set in a Hilbert space
}

\section{BIAGIO RICCERI}

Here and in what follows, $(H,\langle\cdot, \cdot\rangle)$ is a real Hilbert space and $X$ is a non-empty closed convex subset of $H$.

For each $x \in H$, we denote by $P(x)$ the metric projection of $x$ on $X$, that is the unique global minimum of the restriction of the functional $y \rightarrow\|x-y\|$ to $X$.

There is no doubt that the map $P$ is among the most important and studied ones within convex analysis, functional analysis and optimization theory.

For the above reason, we think that it is of interest to highlight some properties of $P$ which do not appear in the wide literature concerning $P$.

We collect such properties in Theorems 1, 2 and 3 below.

First, we fix some notations.

For each $r>0$, we put

$$
B_{r}=\left\{x \in H:\|x\|^{2}<r\right\}
$$

and

$$
S_{r}=\left\{x \in H:\|x\|^{2}=r\right\} .
$$

Moreover, for each $x \in X$, we set

$$
J(x)=\frac{1}{2}\left(\|x\|^{2}-\|x-P(x)\|^{2}+\|P(0)\|^{2}\right) .
$$

Furthermore, for each $r>0$, we put

$$
\gamma(r)=\inf _{x \in S_{r}}\|x-P(x)\|^{2}
$$

Finally, since $P$ is non-expansive in $H$, for each $\lambda \in]-1,1[$, the map $\lambda P$ is a contraction and hence has a unique fixed point that we denote by $\hat{y}_{\lambda}$.

THEOREM 1. - Assume that $0 \notin X$.

Then, the following assertions hold:

$\left(c_{1}\right)$ the function $\lambda \rightarrow g(\lambda):=J\left(\hat{y}_{\lambda}\right)$ is increasing in $]-1,1[$ and its range is $]-\|P(0)\|^{2},\|P(0)\|^{2}[$;

$\left(c_{2}\right)$ for each $\left.r \in\right]-\|P(0)\|^{2},\|P(0)\|^{2}\left[\right.$, the point $\hat{x}_{r}:=\hat{y}_{g^{-1}(r)}$ is the unique point of minimal norm of $J^{-1}(r)$ towards which every minimizing sequence in $J^{-1}(r)$, for the norm, converges ;

$\left(c_{3}\right)$ the function $r \rightarrow \hat{x}_{r}$ is continuous in $]-\|P(0)\|^{2},\|P(0)\|^{2}[$;

$\left(c_{4}\right)$ the function $\lambda \rightarrow h(\lambda):=\left\|\hat{y}_{\frac{1}{\lambda}}\right\|^{2}$ is decreasing in $] 1,+\infty[$ and its range is $] 0,\|P(0)\|^{2}[$;

$\left(c_{5}\right)$ for each $\left.r \in\right] 0,\|P(0)\|^{2}\left[\right.$, the point $\hat{v}_{r}:=\hat{y}_{\frac{1}{h^{-1}(r)}}$ is the unique global maximum of $J_{\mid S_{r}}$ towards which every maximizing sequence for $J_{\mid S_{r}}$ converges ;

$\left(c_{6}\right)$ the function $r \rightarrow \hat{v}_{r}$ is continuous in $] 0,\|P(0)\|^{2}[$.

Assuming, in addition, that $X$ is compact, the following assertions hold:

$\left(c_{7}\right)$ the function $\gamma$ is $C^{1}$, decreasing and strictly convex in $] 0,\|P(0)\|^{2}[$;

$\left(c_{8}\right)$ one has

$$
P\left(\hat{v}_{r}\right)=-\gamma^{\prime}(r) \hat{v}_{r}
$$

for all $r \in] 0,\|P(0)\|^{2}[$;

$\left(c_{9}\right)$ one has

$$
\gamma^{\prime}(r)=-h^{-1}(r)
$$


for all $r \in] 0,\|P(0)\|^{2}[$.

PROOF. Clearly, the set of all fixed points of $P$ agrees with $X$. Now, fix $u \in H$ and $\lambda<1$. We show that

$$
P(u+\lambda(P(u)-u))=P(u) .
$$

If $u \in X$, this is clear. Thus, assume $u \notin X$ and hence $P(u) \neq u$. Let $\varphi: H \rightarrow \mathbf{R}$ be the continuous linear functional defined by

$$
\varphi(x)=\langle P(u)-u, x\rangle
$$

for all $x \in H$. Clearly, $\|\varphi\|_{H^{*}}=\|P(u)-u\|$. We have

$$
\operatorname{dist}\left(u+\lambda(P(u)-u), \varphi^{-1}(\varphi(P(u)))=\frac{|\varphi(u+\lambda(P(u)-u))-\varphi(P(u))|}{\|\varphi\|_{H^{*}}}=(1-\lambda)\|P(u)-u\| .\right.
$$

Moreover, by a classical result ([6], Corollary 25.23), we have

$$
\langle P(u)-u, P(u)-x\rangle \leq 0
$$

for all $x \in X$, that is

$$
X \subseteq \varphi^{-1}([\varphi(P(u)),+\infty[)
$$

Also, notice that

$$
\operatorname{dist}\left(u+\lambda(P(u)-u), \varphi^{-1}(\varphi(P(u)))\right)=\operatorname{dist}\left(u+\lambda(P(u)-u), \varphi^{-1}([\varphi(P(u)),+\infty[)) .\right.
$$

Indeed, otherwise, it would exist $w \in H$, with $\varphi(w)>\varphi(P(u))$, such that

$$
\|u+\lambda(P(u)-u)-w\|<\operatorname{dist}\left(u+\lambda(P(u)-u), \varphi^{-1}(\varphi(P(u))) .\right.
$$

Then, since $\varphi(u+\lambda(P(u)-u))<\varphi(P(u))$ (indeed $\left.\varphi(u+\lambda(P(u)-u))-\varphi(P(u))=(\lambda-1)\|P(u)-u\|^{2}\right)$, by connectedness and continuity, in the open ball centered at $u+\lambda(P(u)-u)$, of radius $\operatorname{dist}(u+\lambda(P(u)-$ $u), \varphi^{-1}(\varphi(P(u)))$, it would exists a point at which $\varphi$ takes the value $\varphi(P(u))$, which is absurd. So, (4) holds. Now, from $(2),(3),(4)$, it follows that

$$
(1-\lambda)\|P(u)-u\| \leq \operatorname{dist}(u+\lambda(P(u)-u), X) \leq\|u+\lambda(P(u)-u)-P(u)\|=(1-\lambda)\|P(u)-u\|
$$

which yields (1). From (1), in particular, we infer that $P(0)=P(-P(0))$. On the other hand, if $\tilde{x} \in H$ is such that $\tilde{x}=-P(\tilde{x})$, then, applying (1) with $u=\tilde{x}$ and $\lambda=\frac{1}{2}$, we get $P(0)=P(\tilde{x})$ and so $\tilde{x}=-P(0)$. Therefore, $-P(0)$ is the unique fixed point of $-P$. Now, let us recall that $J$ is a Fréchet differentiable convex functional whose derivative is equal to $P$ ([1], Proposition 2.2). This allows us to use the results of [3]. Therefore, $\left(c_{1}\right),\left(c_{2}\right),\left(c_{3}\right)$ follow respectively from $\left(a_{1}\right),\left(a_{2}\right),\left(a_{3}\right)$ of Theorem 3.2 of [3], since (with the notation of that result) we have $\eta_{1}=J(-P(0))=-\|P(0)\|^{2}$ and $\theta_{1}=\inf _{X} J=\|P(0)\|^{2}$, while $\left(c_{4}\right),\left(c_{5}\right)$, $\left(c_{6}\right)$ follow respectively from $\left(b_{1}\right),\left(b_{2}\right),\left(b_{3}\right)$ of Theorem 3.3 of [3], since $\theta_{2}=\|P(0)\|^{2}$. Now, assume that $X$ is also compact. Then, $J$ turns out to be sequentially weakly continuous ([5], Corollary 41.9). Moreover, $J$ has no local maxima since $P$ has no zeros. At this point, $\left(c_{7}\right),\left(c_{8}\right),\left(c_{9}\right)$ follow respectively from $\left(b_{4}\right),\left(b_{5}\right)$, $\left(b_{6}\right)$ of Theorem 3.3 of [3], since, for a constant $k_{0}$, we have

$$
\sup _{S_{r}} J=-\frac{1}{2} \gamma(r)+k_{0}
$$

for all $r>0$. The proof is complete.

THEOREM 2. - Let $Q: H \rightarrow H$ be a continuous and monotone potential operator such that

$$
\lim _{\|x\| \rightarrow+\infty} I(x):=\int_{0}^{1}\langle Q(s x), x\rangle d s=+\infty .
$$


Set

$$
\lambda^{*}=\inf _{r>\inf _{H}} \inf _{I x \in I^{-1}(]-\infty, r[)} \frac{J(x)-\inf _{\left.\left.y \in I^{-1}(]-\infty, r\right]\right)} J(y)}{r-I(x)} .
$$

Then, the equation

$$
P(x)+\lambda Q(x)=0
$$

has a solution in $H$ for every $\lambda>\lambda^{*}$. Moreover, when $\lambda^{*}>0$, the same equation has no solution in $H$ for every $\lambda<\lambda^{*}$.

PROOF. Since $Q$ is a monotone potential operator, the functional $I$ turns out be convex, of class $C^{1}$ and its derivative agrees with $Q$. Now, the conclusion follows from Theorem 2.4 of [2], since, by convexity, the solutions of the equation $P(x)+\lambda Q(x)$ are exactly the global minima in $H$ of the functional $J+\lambda I . \triangle$

THEOREM 3. - Let $(T, \mathcal{F}, \mu)$ be a measure space, with $0<\mu(T)<+\infty$ and assume that $0 \notin X$.

Then, for every $\eta \in L^{\infty}(T)$, with $\eta \geq 0$, for every $\left.r \in\right] 0,\|P(0)\|^{2}[$ and for every $p \geq 2$, if we put

$$
U_{\eta, r}=\left\{u \in L^{p}(T, H): \int_{T} \eta(t)\|u(t)\|^{2} d \mu=r \int_{T} \eta(t) d \mu\right\},
$$

we have

$$
\inf _{u \in U_{\eta, r}} \int_{T} \eta(t)\|u(t)-P(u(t))\|^{2} d \mu=\inf _{x \in S_{r}}\|x-P(x)\|^{2} \int_{T} \eta(t) d \mu
$$

and

$$
\sup _{u \in U_{\eta, r}} \int_{T} \eta(t)\|u(t)-P(u(t))\|^{2} d \mu=\sup _{x \in S_{r}}\|x-P(x)\|^{2} \int_{T} \eta(t) d \mu .
$$

PROOF. Applying Theorem 5 of [4] to $J$ and $-J$, respectively, we obtain

$$
\inf _{u \in V_{\eta, r}} \int_{T} \eta(t) J(u(t)) d \mu=\inf _{S_{r}} J \int_{T} \eta(t) d \mu
$$

and

$$
\sup _{u \in V_{\eta, r}} \int_{T} \eta(t) J(u(t)) d \mu=\sup _{S_{r}} J \int_{T} \eta(t) d \mu
$$

where

$$
V_{\eta, r}=\left\{u \in L^{p}(T, H): \int_{T} \eta(t)\|u(t)\|^{2} d \mu \leq r \int_{T} \eta(t) d \mu\right\} .
$$

Now, observe that $J_{\mid S_{r}}$ has a global minimum. Indeed, since $J$ is weakly lower semicontinuous and $\overline{B_{r}}$ is weakly compact, $J_{\mid \overline{B_{r}}}$ has a global minimum, say $\hat{w}_{r}$. Notice that $\hat{w}_{r} \in S_{r}$, since, otherwise, $P\left(\hat{w}_{r}\right)=0$ which is impossible since $0 \notin X$. So, $\hat{w}_{r}$ is a global minimum of $J_{\mid S_{r}}$. Furthermore, from Theorem 1, we know that $J_{\mid S_{r}}$ has a global maximum, say $\hat{v}_{r}$. Denote by the same symbols the constant functions (from $T$ into $Y$ ) taking, respectively, the values $\hat{w}_{r}$ and $\hat{v}_{r}$. Since $\mu(T)<+\infty$, we have $\hat{w}_{r}, \hat{v}_{r} \in U_{\eta, r}$. So, from (7) and (8), it follows respectively

$$
\inf _{u \in V_{\eta, r}} \int_{T} \eta(t) J(u(t)) d \mu=\int_{T} J\left(\hat{w}_{r}\right) \eta(t) d \mu \geq \inf _{u \in U_{\eta, r}} \int_{T} \eta(t) J(u(t)) d \mu
$$

and

$$
\sup _{u \in V_{\eta, r}} \int_{T} \eta(t) J(u(t)) d \mu=\int_{T} J\left(\hat{v}_{r}\right) \eta(t) d \mu \leq \sup _{u \in U_{\eta, r}} \int_{T} \eta(t) J(u(t)) d \mu .
$$

Therefore

$$
\inf _{S_{r}} J \int_{T} \eta(t) d \mu=\left(r+\|P(0)\|^{2}-\sup _{x \in S_{r}}\|x-P(x)\|^{2}\right) \int_{T} \eta(t) d \mu=\inf _{u \in U_{\eta, r}} \int_{T} \eta(t)\left(\|u(t)\|^{2}-\|u(t)-P(u(t))\|^{2}+\|P(0)\|^{2}\right) d \mu
$$




$$
=\left(r+\|P(0)\|^{2}\right) \int_{T} \eta(t) d \mu-\sup _{u \in U_{\eta, r}} \int_{T} \eta(t)\|u(t)-P(u(t))\|^{2} d \mu
$$

which yields (6). Likewise

$$
\begin{gathered}
\sup _{S_{r}} J \int_{T} \eta(t) d \mu=\left(r+\|P(0)\|^{2}-\inf _{x \in S_{r}}\|x-P(x)\|^{2}\right) \int_{T} \eta(t) d \mu=\sup _{u \in U_{\eta, r}} \int_{T} \eta(t)\left(\|u(t)\|^{2}-\|u(t)-P(u(t))\|^{2}+\|P(0)\|^{2}\right) d \mu \\
=\left(r+\|P(0)\|^{2}\right) \int_{T} \eta(t) d \mu-\inf _{u \in U_{\eta, r}} \int_{T} \eta(t)\|u(t)-P(u(t))\|^{2} d \mu
\end{gathered}
$$

which yields (5) 


\title{
References
}

[1] S. FITZPATRICK and R. PHELPS, Differentiability of the metric projection in Hilbert space, Trans. Amer. Math. Soc., 270 (1982), 483-501.

[2] B. RICCERI, A general variational principle and some of its applications, J. Comput. Appl. Math., 113 (2000), 401-410.

[3] B. RICCERI, Fixed points of nonexpansive potential operators in Hilbert spaces, Fixed Point Theory Appl. 2012, 2012: 123.

[4] B. RICCERI, Integral functionals on $L^{p}$-spaces: infima over sub-level sets, Numer. Funct. Anal. Optim., 35 (2014), 1197-1211.

[5] E. ZEIDLER, Nonlinear Functional Analysis and its Applications, vol. III, Springer-Verlag, 1985.

[6] E. ZEIDLER, Nonlinear Functional Analysis and its Applications, vol. II/B, Springer-Verlag, 1990.

\author{
Department of Mathematics \\ University of Catania \\ Viale A. Doria 6 \\ 95125 Catania, Italy \\ e-mail address: ricceri@dmi.unict.it
}

